\title{
Traduire
}

Une eatre perspective sur $r$ tatadcicion

Revue française de la traduction

$226 \mid 2012$

Face au miroir

\section{Une traduction « puissance trois » : Rachid Djaïdani et la langue des cités}

Problématiques et stratégies de traduction dans le contexte italien

Ilaria Vitali

\section{OpenEdition}

\section{Journals}

Édition électronique

URL : http://journals.openedition.org/traduire/165

DOI : 10.4000/traduire.165

ISSN : 2272-9992

\section{Éditeur}

Société française des traducteurs

Édition imprimée

Date de publication : 1 janvier 2012

Pagination : 108-119

ISBN : 039-773X

ISSN : 0395-773X

\section{Référence électronique}

Ilaria Vitali, «Une traduction « puissance trois » : Rachid Djaïdani et la langue des cités », Traduire [En ligne], 226 | 2012, mis en ligne le 03 février 2014, consulté le 10 octobre 2020. URL : http:// journals.openedition.org/traduire/165; DOI : https://doi.org/10.4000/traduire.165 


\section{Une traduction « puissance trois » : Rachid Djaïdani et la langue des cités}

Problématiques et stratégies de traduction dans le contexte italien Ilaria Vitali

\section{NOTE DE L'ÉDITEUR}

http://www2.lingue.unibo.it/francofone/paginavitali.htm

1 Au tournant du nouveau millénaire, le «français des cités $»^{1}$, parler codé né dans les quartiers extra muros des grandes villes françaises, est devenu un langage littéraire de plus en plus exploité par de nombreux écrivains. Pour s'en rendre compte, il suffit de penser aux ouvrages de quelques écrivains " urbains " ${ }^{2}$, dont Faïza Guène, Mabrouck Rachedi ou Rachid Djaïdani, pour ne citer qu'eux. La stratification langagière qui paraît dans leurs ouvrages ne se limite pas à la reproduction d'un sociolecte qui caractérise un groupe de personnages "marginaux », mais devient une véritable stratégie littéraire utilisée consciemment. En exploitant l'extrême richesse créative offerte par ce nouveau langage, les écrivains en font un usage "actif» et emploient ses procédés de (dé)formation lexicale pour faire œuvre d'art. Il ne s'agit pas, pour eux, de reproduire de manière stérile une langue-témoignage dans une visée documentaire, mais de «bricoler " la matière verbale, en faisant éclater les frontières du français standard en même temps que celles de l'œuvre narrative.

Ce procédé de dynamisation - et « dynamitation » - linguistique et littéraire fortifie les textes des auteurs urbains, mais contribue en même temps à les rendre apparemment « intraduisibles ». Et pourtant, ces écrivains extra muros dépassent de plus en plus les frontières de l'Hexagone. On pense, pour se limiter à quelques exemples, aux traductions allemandes d'Azouz Begag ${ }^{3}$, à la traduction norvégienne du premier roman de Mohamed Razane ${ }^{4}$, ou bien à la traduction de Kiffe kiffe demain de Faïza Guène dans 
plus de vingt pays. Tout traducteur qui s'approche du roman « urbain» aura toutefois affaire à des textes qui demandent un effort interprétatif important et, qui plus est, résistent - du moins à première vue - aux tentatives de traduction.

En m'appuyant sur mon expérience en tant que traductrice italienne de quelques-uns de ces écrivains ${ }^{5}$, je propose un tour d'horizon des défis traductologiques que posent ces textes. Pour ce faire, je restreindrai l'analyse aux romans de l'écrivain d'origine algérienne Rachid Djaïdani ${ }^{6}$, qui me paraissent particulièrement représentatifs. Dans cet article, il sera question des démarches et stratégies traductives qu'on peut mettre en œuvre dans un contexte littéraire hybride ${ }^{7}$, pour voir de quelle manière la réflexion traductologique peut aider à éclairer les enjeux linguistiques et littéraires de ces textes.

\section{Argot classique, verlan, xénolectes : les entorses à la langue normative du " français des cités »}

4 Contrairement à l'image souvent reproduite par les médias, les cités des banlieues françaises ne sont pas seulement des «non-lieux au procès des bandes de la société bonne et bien $»^{8}$. Elles sont aussi une réalité faite de codes d'honneur, de rituels de communication, de performances artistiques et surtout langagières. Comme tout langage argotique, celui qui s'est développé dans les cités a initialement pour fonction de crypter le message, d'exclure tout tiers de la communication, notamment «les autorités »- policiers, professeurs et parents -, les locuteurs étant souvent très jeunes. S'il existe dans la langue actuelle ce qu'on appelle "l'argot commun ", à savoir un ensemble de mots et de tournures qui, au cours des siècles, se sont infiltrés dans le français normatif et dont l'emploi relève du choix de registres du locuteur, le « français des cités " garde encore, pour sa part, des fonctions cryptiques. On ne saurait toutefois insister assez sur la deuxième fonction que recouvre ce sociolecte, à savoir la fonction identitaire. Ce langage permet en effet la reconnaissance mutuelle des membres du groupe, et proclame dans le même temps leur séparation de la société, car "plus un groupe est ou se veut différent, plus il aura tendance à utiliser des formes linguistiques différentes $»^{10}$. Aujourd'hui cependant, l'aspect le plus important de ce langage semble s'illustrer à travers ce qu'il a de ludique. Bricolés et traficotés, les mots du «français des cités» permettent de jouer avec la langue normative et sont vécus comme un « exercice d'évasion » par ceux qui l'emploient ${ }^{11}$.

Mais comment traduire ce langage crypto-ludique?

6 Dans le cas des romans de Rachid Djaïdani, le premier défi traductologique concerne sans aucun doute la reproduction des pratiques verlanesques, inexistantes dans la plupart des langues européennes. On entend par verlan un argot à clef dont le procédé d'encodage fixe consiste à inverser les syllabes phonétiques des mots (dans le cas le plus simple: S1S2 > S2S1, ex. "cité » > " téci »), le mot "verlan » étant lui même la verlanisation de « (à) l'envers ». Malgré ce que l'on aurait tendance à croire, il ne s'agit pas d'un procédé nouveau, le verlan ayant déjà été à l'œuvre au XVI siècle, quoique de manière limitée. Après une vie plus ou moins souterraine, il s'est répandu après la Seconde Guerre mondiale, pour connaitre ensuite un développement énorme dans les banlieues défavorisées, notamment à partir des années 1970 et 1980. Si le verlan représente aujourd'hui le langage d'une génération, d'un groupe social et d'un espace urbain spécifiques, il est aussi un instrument de subversion en ce qu'il épouse parfaitement les enjeux contestataires du texte «urbain»: derrière l'inversion 
syllabique il est facile d'entrevoir le renversement de la langue normative, mais aussi celui d'un système de valeurs et d'une identité franco-française dans lesquels les locuteurs ne se reconnaissent souvent pas. Chez Djaïdani, les nombreuses occurrences de verlan contribuent à dynamiter la « langue officielle »: des mots tels que « téma » (< mater), « chelou » (< louche), keuf ( $<$ flic), beur (< arabe) $)^{12}$, etc. sont très courants dans ses romans où ils témoignent de l'effervescence linguistique à l'œuvre dans les cités.

Il faut remarquer aussi le fait que, dans le «français des cités », le verlan est associé à des variantes diatopiques, donc des emprunts, des xénismes, des formes hybrides. Cette hybridation linguistique a pour but de «traduire » une réflexion (post)identitaire qui se bâtit à partir d'une mosaïque de pièces multiples. C'est sans doute pour cela que les auteurs urbains cultivent systématiquement dans leurs romans une autre pratique courante dans le langage des cités, le code switching, à savoir l'alternance intraphrasique de termes français et allogènes, qui devient un véritable manifeste du métissage culturel et identitaire. Les différents aspects du vécu langagier de ces auteurs de la post-migration, ainsi que l'ensemble des contacts linguistiques vécus depuis l'enfance dans des cités multiethniques, ont une influence directe sur le développement de la création d'un langage vernaculaire, agrégat instable d'emprunts. Si la langue des romans de Rachid Djaïdani - et de beaucoup d'autres - voit d'abord la coprésence de deux idiomes (le français et la langue parlée à l'origine par les parents, le darija), on doit reconnaître que l'alternance codique se fait désormais entre plusieurs langues, ce qui s'explique facilement en tenant compte du melting-pot des banlieues actuelles. Véhiculant une dynamique multiculturelle, la cohabitation plurilinguistique crée ainsi des textes-palimpsestes.

8 Compte tenu de tous ces éléments, il est facile de comprendre que le risque principal pour un traducteur qui aborde ce genre de textes est celui de les «neutraliser», en laissant de côté leur stratification et leur ramification plurilinguistique. Bref, de céder aux tendances défor- mantes identifiées par Berman comme la «destruction des réseaux vernaculaires " et l'« effacement des superpositions des langues »"

9 Pour commencer son travail, le traducteur peut consulter les autres traductions déjà présentes dans le même domaine, ainsi que les textes littéraires produits, dans la langue cible, par des écrivains migrants. Dans le cas spécifique du contexte italien, il faut avouer que les écritures migrantes sont un phénomène tout à fait récent (on a fêté en 2010 les vingt ans de leur naissance), surtout si l'on considère uniquement les ouvrages produits par les auteurs de la «deuxième génération » de l'immigration, qui correspondraient, en quelque sorte, au statut de Rachid Djaïdani. La jeunesse de cette littérature "italophone» a des conséquences d'ordre stylistique et linguistique, à commencer par une forme de respect exaspéré pour la langue normative (en écrivant, les « immigrés » semblent vouloir prouver leur maîtrise de la langue du pays d'accueil). Bref, pour le dire, non sans une certaine ironie, avec les mots d'Ortega y Gasset: ces écrivains semblent craindre « l'apparat policier de la grammaire ». Or, Djaïdani attaque volontiers cet apparat.

10 Par ailleurs, pour ce qui est des autres traductions liées au contexte migratoire déjà réalisées en italien, il faut avouer qu'il y a une certaine "insularité ", chaque traducteur procédant à ses propres choix (motivés par le contexte éditorial, par le public cible, etc.), apparemment sans véritable confrontation sur les stratégies que l'on peut tour à tour mettre en œuvre. Cela s'explique sans doute dans la mesure où il s'agit d'un phénomène assez récent, surtout si on se limite aux seuls textes, très peu traduits, 
qui jouent sur le « français des cités $»^{14}$. Dans ce cas, les dictionnaires bilingues n'aident pas le traducteur, l'obligent à des prouesses, pirouettes et autres acrobaties. Bref, à une véritable gymnastique langagière. Cela est dû au fait que les dictionnaires, d'une part, peinent à suivre le mouvement très rapide de la langue, d'autre part, ne tiennent souvent pas assez compte des marques sociolinguistiques dans la traduction qu'ils proposent $t^{15}$. Si je devais décrire ma traduction des ouvrages de Djaïdani, j'emploierais donc, sans hésitation, l'expression «traduction puissance trois » : à savoir du «français des cités " au français standard, du français standard à l'italien standard, de l'italien standard à une forme particulière d'italien argotique. Le risque d'un tel processus de traduction "puissance trois » est évident : celui de s'arrêter à la " puissance deux », et donc d'opérer une normalisation du texte - la destruction des réseaux vernaculaires de Berman - en aplatissant ses « aspérités ». Plus que jamais, le traducteur qui s'évertue à ces acrobaties langagières doit veiller à compléter son " exercice gymnique », et à ne pas se laisser intimider par « l'apparat policier de la grammaire».

11 Pour traduire le langage des romans de Rachid Djaïdani, j'ai donc puisé dans les couches de la langue parlée, en l'émaillant de nombreuses expressions reprises de l'argot des jeunes, notamment pour reproduire le verlan ${ }^{16}$. En effet, les narrateurs ainsi que les locuteurs de Djaïdani sont de préférence très jeunes, et plusieurs lexèmes employés dépassent d'ailleurs les banlieues et touchent la jeunesse toutes origines confondues. Cela a tout de même posé certains problèmes, car en Italie, à la différence de ce qui se passe en France, le langage des jeunes change radicalement de ville en ville. Plusieurs choix lexicaux ont d'ailleurs fait l'objet d'une "médiation" entre mes propositions de formes argotiques - utilisées de préférence dans l'Italie du Nord, où je réside - et celles de l'éditeur Giulio Perrone à Rome.

Pour reproduire le langage de Djaïdani, j'ai essayé de créer tout d'abord un argot commun qui puisse traduire la koiné des jeunes, reposant notamment sur un certain imaginaire télévisuel, para-littéraire et musical, tout en évitant les éléments dialectaux trop marqués ${ }^{17}$. En ce sens, Internet s'est révélé aussi un allié précieux. J'ai ensuite puisé dans les codes linguistiques des rappeurs, des crews, bref, du monde du hip hop italien, pour reproduire plusieurs mots du verlan, dont, par exemple, le mot «tipa ", employé par les rappeurs dans le sens de "jeune femme ", voire "fiancée », que j'ai utilisé pour traduire le mot «meuf » dans différents contextes. La musique rap m’a été également utile pour représenter une certaine virulence verbale, frisant la scatologie, qui caractérise particulièrement le «français des cités ».

13 La reproduction de ce parler jeune n'était toutefois pas suffisante, car le langage de Djaïdani (et de nombreux autres écrivains "urbains») est un parler vernaculaire interethnique où l'on perçoit bien l'osmose de langues et de cultures, fruit de la postmigration. Reproduire l'hétérolinguisme constitue donc un autre défi majeur dans la traduction de ce genre de textes. À ce sujet, Berman avait exprimé son scepticisme dès $1984^{18}$ quant à la possibilité de trouver une traduction convenable aux textes qui présentent un sous-bois linguistique hybride. Pour rendre la polyphonie langagière, il est en effet indispensable de reproduire le métissage langagier, avec sa stratification d'emprunts et de variantes qui, par la superposition, donne une dimension de profondeur aux textes. Dans ma traduction des romans de Djaïdani, les termes arabes ainsi que les autres xénismes ont été conservés, en adoptant la translittération italienne quand c'était nécessaire. À côté des mots allogènes, je n'ai utilisé que rarement les explicitations ou les développements définitionnels, préférant insérer, 
notamment dans Viscéral ${ }^{19}$, un glossaire à la fin du texte pour ne pas couper le rythme de la lecture, sans pour autant priver le lecteur de l'explication de mots problématiques. Pendant ma traduction, j'ai d'ailleurs découvert que certaines expressions du darija reprises dans le «français des cités » sont également exploitées par les quelques jeunes auteurs "urbains» italiens (par exemple, la formule «Wesh?»/« Wesh khoyya? » qui ouvre les rituels complexes de salutation ${ }^{20}$ ).

Pour ce qui est des emprunts à l'anglais qui imprègnent la prose de Djaïdani et de bien d'autres auteurs « urbains ", la question mérite quelques précisions supplémentaires. À la différence du français, l'italien accueille en effet volontiers des termes anglais dans la langue courante et cela n'est d'ailleurs pas perçu comme une subversion de la «langue légitime ». J'ai donc insisté sur les anglicismes, pour rendre cette tendance de l'auteur qui risquait autrement de passer presque inaperçue ${ }^{21}$.

Autre problème, la reproduction du xénolecte qui caractérise le parler des personnages immigrés, notamment arabes (par exemple les parents des personnages "beurs»), maitrisant mal le français. À partir de quelques mots qui reproduisent des connaissances linguistiques vagues insérées dans un texte tout à fait compréhensible, on peut arriver à des phrases entières qui visent à imiter graphiquement certaines distorsions langagières. L'exploitation littéraire du franco-arabe parlé par les immigrés constitue chez Djaïdani un véritable instrument narratif qui aboutit à des effets de style hautement comiques.

Pour traduire en italien ce genre de xénolecte, certains préconisent le recours aux variantes dialectales de l'Italie méridionale, ce qui reproduirait un décalage avec la langue normative. Dans son étude sur les choix linguistiques et traductologiques d'un roman du marocain Driss Chraïbi, Naissance à l'aube, Francesco Paolo Alexandre Madonia propose, par exemple, l'emploi d'un italien du centre-sud mâtiné de mots empruntés au dialecte sicilien pour traduire le "français métissé » des personnages maghrébins qui maîtrisent mal le français ${ }^{22}$. L'emploi de ces mots, qui a souvent pour effet de produire un aboutissement comique, suivrait la formule bien rodée, entre autres, des romans d'Andrea Camilleri. Quoiqu'intéressante, cette stratégie ne pouvait, à mon sens, s'appliquer aux romans de Djaïdani. J'ai donc choisi d'adapter le « français cassé » en créant une sorte de pseudo-italien métissé, qui reproduit une pratique approximative de la langue à travers l'emploi de constructions «fautives», de verbes uniquement à l'infinitif ou d'approximations phonétiques dues aux interférences. En voici quelques exemples : « Ils sont friches, ils sont bounnes, mi pîches [...] Si la viriti... » ${ }^{23}$; «Essere fresche, essere buone, le mie pesche [...] Essere la verità... $»^{24}$; et encore " oui misieu, si vi pli, ti di suite patron $»^{25} ;$ « sì monsiù, grazie mille, subido badrone $»^{26}$.

\section{Au-delà du roman : cinélangue et slam}

$17 \mathrm{Au}$ niveau stylistique, ce sont le tressage générique et la multimédialité qui caractérisent les romans de Djaïdani. Si la littérature « urbaine » se compose presque entièrement de romans, il faut préciser que les auteurs en poussent les limites jusqu'à les faire éclater. Cette écriture, qui mélange des pratiques scripturaires hétéroclites, où le rythme est ultra-rapide, syncopé, à l'image d'une société « liquide » à la Zygmunt Bauman, est la bannière des écrivains «urbains». Au sein de ces textes, plusieurs médias se côtoient, se font écho, se fécondent réciproquement. Les frontières entre les genres s'estompent, ainsi que celles entre médias expressifs. Poèmes et morceaux de 
musique, références au cinéma et à Internet, créent ainsi des ouvrages multidimensionnels. Intervient ici, entre autres, la pratique du slam. Poésie " urbaine » importée des États-Unis, le slam a pour but de «claquer » l'auditeur avec les mots, de l'attraper par le son et les images, et se rapproche parfois de la véritable performance, car ces textes sont souvent faits pour être lus à voix haute ${ }^{27}$. Bien que le renvoi à la culture nord- américaine soit évident (le slam serait né en 1984 dans les clubs de Chicago), on y entrevoit une tradition de joutes oratoires très ancienne et bien française - on pense aux tençons des troubadours. C'est peut-être pour cela que le slam s'est greffé rapidement en France, où des éléments "francophones " (inspirés, par exemple, des pratiques des griots de l'Afrique sub-saharienne) ont été introduits par la suite.

Dans le cas des romans de Rachid Djaïdani, les phrases suivent parfois une métrique précise qui respecte, malgré l'auteur (qui a avoué ne pas être conscient de ce procédé) la versification classique française : certaines phrases sont des alexandrins, d'autres des octosyllabes, vers par excellence de la musique rap. Par ailleurs, les romans de l'auteur sont émaillés de rimes, d'allitérations, d'onomatopées qui créent des échos phonétiques. Djaïdani use et abuse de mots aux signifiants proches et aux signifiés divergents, en mettant en œuvre assonances et paronomases. Des jeux sonores comme «tiges de quinze piges $»^{28}$, « smail sans émail ${ }^{29}$, « marécage sans âge $»^{30}$ étoilent les pages de Djaïdani, qui dépasse par ces résonances le seuil du loufoque pour catalyser les effets de sens. L'emploi des homéotéleutes ou bien des allitérations crée des effets rythmiques particuliers notamment dans Mon ne ${ }^{\circledR}$, où l'auteur s'en sert, entre autres, pour montrer la lenteur du temps qui passe par un savant emploi des consonnes liquides (par exemple, dans le passage « l'heure coule et s'écoule ${ }^{31}$ ).

Le traducteur se doit d'essayer de garder ce côté sonore ainsi que la musicalité du texte en prose, en évitant autant que possible la « destruction des rythmes » (Berman). J'ai veillé à garder autant que possible ces effets sonores dans ma traduction, en exploitant, si nécessaire, des stratégies de compensation. Dans certains cas, un glissement s'est produit : par exemple, j'ai dû remplacer la liquide « 1 » du passage cité ci-dessus par « $\mathrm{r} »(«$ l'ora gira e rigira ») ou encore la rime de «smail sans émail » est devenue en italien une allitération («smail senza smalto »).

Pour trouver le bon rythme de la narration, la musique rap italienne écoutée pendant ma traduction s'est effectivement révélée un outil précieux, tout comme la consultation de l'anthologie du rap italien, Potere alla parola, éditée par le chanteur Jovanotti ${ }^{32}$. En effet, c'est dans la musique rap que j'ai pu trouver plusieurs solutions traductives intéressantes : je me limite à citer ici le néologisme/jeu de langage "pu-pulzella », tiré d'une chanson du groupe Articolo 31, que j'ai choisi pour traduire la « fée-melle » du roman Mon ne ${ }^{\circledR}$ de Rachid Djaïdani.

21 Si l'écriture de cette nouvelle génération d'écrivains « urbains » se fait de plus en plus sonore, elle est aussi visuelle, au point qu'on pourrait parler d'une véritable cinélangue. Il n'est sans doute pas anodin qu'une bonne partie des écrivains « urbains » ait aussi des expériences de scénaristes, de réalisateurs, voire d'acteurs. En effet, la langue de Djaïdani est "cinématographique »: non seulement elle doit beaucoup à l'image filmique en termes d'inspiration et de stratégies d'écritures (le premier jet de son roman Boumkoeur n'était rien d'autre qu'un scénario), mais elle s'attache à donner à l'écrit une profondeur en trois dimensions par l'emploi massif d'images et de figures de style novatrices. 

fenêtres pour l'œil, qu'il faut garder dans la traduction. Ses romans ne se limitent pas à créer des hypotyposes, mais font fusionner les deux mondes - écriture et image - dans un court-circuit explosif. Il n'est pas inutile de rappeler que Djaïdani s'est filmé pendant l'écriture de son deuxième roman, Mon $n e^{\circledast} f$, pour répondre à l'Establishment littéraire qui l'avait accusé de ne pas écrire lui-même ses ouvrages ${ }^{33}$. Si ce procédé est sans doute perturbant et doublement éloquent dans son enjeu provocateur, il n'est pas difficile d'imaginer que le côté visuel de la création artistique ait influencé l'écriture de l'œuvre narrative elle-même. L'importance de l'aspect visuel se lit dès le titre de ce roman; en effet, le symbole ${ }^{\oplus}$, censé représenter le R.E.R. où se déroule l'action, peut aussi être lu au sens de marque déposée, et donc gage d'authenticité de l'auteur qui en fait usage. D'ailleurs, les nombreuses onomatopées utilisées par l'auteur, en particulier dans Mon $n e^{\oplus} f$, ne constituent pas uniquement des refrains musicaux qui émaillent la narration, mais apparentent le roman à une véritable bande dessinée: la graphie différente ainsi que le caractère plus gros employé alors mettent d'autant mieux en évidence l'importance que l'auteur accorde à la fusion du sonore et du visuel.

En ce qui concerne la traduction des onomatopées, je n'ai eu qu'à adopter l'italien. Au contraire, pour ce qui est du titre du roman, polysémique et difficilement traduisible (Mon $n e^{\otimes} f$, mais aussi Mon air ou Mon R), l'éditeur a finalement choisi de le changer complètement (Ritratto di un ragazzo da buttare alle or tiche). J'avoue que cette solution ne m'a jamais convaincue, et que j'aurais préféré un titre court et précis qui jouerait sur le champ sémantique du hip hop, par exemple Freestyler (le narrateur se définit ainsi dans son monologue fiévreux) ou bien Underground, qui aurait non seulement évoqué le monde d'une certaine sub-culture urbaine, mais aussi l'univers souterrain du R.E.R. dans lequel se déroule, répétons-le, l'histoire.

Un autre exemple intéressant de fusion entre les aspects sonore et visuel chez Djaïdani est fourni par un tag dans le hall de la tour H.L.M. de Lies, héros du roman Viscéral. On y lit l'inscription: «Mon hall ma T'hall mes pole positions $»^{34}$. Dans ce graffiti, les mots « hall», «T'hall» (taule) et " pole» riment tous en [ol]. Située au centre de la page (donc cruciale sur le plan visuel), cette phrase est révélatrice car elle fait référence à deux lieux « initiatiques " pour de nombreux jeunes des cités : le hall des tours et des barres, lieu de rencontre du "clan », et la prison, où beaucoup finissent par passer : « tu n'appartiens pas au clan » poursuit Djaïdani «si tu n'as pas goûté à la gamelle » ${ }^{35}$. Or, il était important de traduire ce tag révélateur en faisant ressortir l'effet rythmique, qui, par l'appât sonore, double et renforce l'aspect visuel. Comme dans d'autres passages du même roman, j'ai traduit " taule » par "gabbio", italien argotique pour " prison ». Pour ne pas perdre la rime, j'ai choisi de traduire « hall» - qui indique les tours H.L.M. et, par extension, l'appartenance à la « zone » - par «suburbio », italien argotique pour «banlieue ». Le tag, en italien, donne cela : « il suburbio il gabbio le mie pole position ». Ce glissement métonymique m'a permis de ne pas perdre complètement la consonance rythmique du tag, tout en gardant ses aspects vernaculaires et « oraculaires » concernant la vie des cités.

Les « glissements » de ce genre, voire les stratégies de compensation qui consistent à transférer ailleurs dans le texte un jeu de mot qu'il n'était pas possible de reproduire au même endroit dans la langue cible, constituent parfois les seules solutions possibles. Pour ces raisons, une traduction devrait toujours être considérée dans son ensemble plutôt que confrontée mot à mot au texte original. 


\section{Conclusion} traductologiques montre à quel point les espaces périphériques des cités semblent avoir toutes les chances de devenir de nouveaux centres de création artistique, l'habitat «concentrationnaire " étant à l'origine d'un langage foisonnant qui invite à questionner le «canon » linguistique et littéraire français. Paradoxalement, la « crise des banlieues ", question sociale majeure au tournant de ce nouveau millénaire, s'est avérée productive pour de nombreux écrivains, dont Djaïdani ne constitue ici qu'une figure d'exemple. Par ailleurs, si le degré d'ancrage dans la sub-culture des cités est très fort dans ces romans, de nombreux thèmes traités par l'auteur (réflexion identitaire à l'ère de la globalisation, hybridisme, multiculturalisme, intégration sociale...) sont autant de questions universelles, qui touchent à l'humain. Cela m'est apparu d'une manière assez évidente dès que j'ai commencé à réfléchir sur les défis de ces textes "métissés » et sur les solutions linguistiques et stylistiques possibles, car la traduction marche comme une sorte de camera obscura, qui reflète et développe les virtualités du texte-source.

Techniques de compensation, apparats paratextuels, développements définitionnels... toutes ces stratégies - et bien d'autres - peuvent s'avérer des instruments utiles au traducteur ; entre ces multiples techniques, il ne faut pourtant jamais oublier d'opérer un travail de (re)négociation constante. Comme l'affirme Keil, la traduction est peutêtre finalement un "cas de figure- modèle de tout écriture, fût-ce dans la langue maternelle, avec un potentiel de sensibilisation extrême, justement, pour l'« étrangeté » inhérente à toute langue, pour sa plasticité, son individualité, sa matérialité $»^{36}$. C'est précisément là, dans cette sensibilisation à l'étrange qui devient proche, que se cache le plaisir de toute traduction.

\section{BIBLIOGRAPHIE}

\section{Sources primaires}

DJAÏDANI Rachid, 2007, Viscéral, Paris, Seuil. (Viscerale, tr. it. Ilaria Vitali, Rome, Giulio Perrone Editore, 2009).

DJAÏDANI, Rachid, 2004, Mon $N e^{\oplus}$, Paris, Seuil. (Ritratto di un ragazzo da buttare alle or tiche, 2011, tr. it. Ilaria Vitali, Rome, Giulio Perrone Editore).

\section{Sources secondaires}

BASSNETT Susan et TRIVEDI Harish, (éd.), 1999, Post-colonial Translation. Theory and Practice, Londres, Routledge.

BERMAN Antoine, 1999, La Traduction et la lettre ou l'auberge du lointain, Paris, Seuil. 
BERMAN Antoine, 1984, L'Épreuve de l'étranger. Culture et traduction dans l'Allemagne romantique, Paris, Gallimard.

BOLLETTIERI BOSINELLI Rosa Maria et DI GIOVANNI Elena (éd.), 2009, Oltre l'occidente. Traduzione e alterità culturale, Milan, Bompiani.

CASANOVA Pascale, 2002, «Consécration et accumulation du capital littéraire : la traduction comme échange inégal », in Actes de la recherche en sciences sociales, vol. 144, p. 7-20.

CAUBET Dominique, 2005, «Ce français qui nous (re)vient du Maghreb : mélanges linguistiques en milieux urbains ", in Cultures Sud, n 159, p. 8-15.

ELEFANTE Chiara, 2004, « Arg. et pop., ces abréviations qui donnent les jetons aux traducteursdialoguistes ", in Meta : journal des traducteurs, vol. 49, n 1, p. 193-207.

GOUDAILLIER Jean-Pierre, 1997, Comment tu tchatches! Dictionnaire du français contemporain des cités , Paris, Maisonneuve et Larose.

GOUDAILLIER Jean-Pierre, 2002, « De l'argot traditionnel au français contemporain des cités », in La linguistique, vol. $38, \mathrm{n}^{\circ}$ 1, p. 5-24.

KEIL-SAGAWE Regina, 2005, « La main de Fatima. Problématique du transfert culturel dans la traduction/réception de littérature maghrébine d'expression française en allemand ", in AÏT EL FERRANE Mohamed, (éd.), Kulturen des Lehrens und Lernens. Dialog der Bildungs - und Erziehungssysteme : Heidelberg - Marrakesch, Marrakech, Publications de la Faculté des Lettres et des Sciences Humaines, El Watanya. Disponible sur le site : www.uebersetzungswissenschaft.de/ keil-Fatima.pdf, consulté le 10 janvier 2012.

LEPOUTRE David, 1997, Cœur de banlieue, Paris, Odile Jacob.

SIMON Sherry, 1995, « La culture transnationale en question : visées de la traduction chez Homi Bhabha et Gayatri Spivak », in Études françaises, vol. 31, n 3, p. 43-57.

\section{NOTES}

1. Langue de la marge, le «français contemporain des cités » s'est développé au cours des années 1970 et 1980 dans les banlieues pauvres, dans la visée d'exprimer une réalité urbaine nouvelle et métissée. Il s'agit d'un sociolecte assez particulier constitué aujourd'hui de plusieurs composantes, de l'argot classique au verlan, jusqu'à la cyberl@ngue (Aurelia Dejond, 2000), à savoir la langue d'Internet ; à cela s'ajoutent de nombreux xénismes, des emprunts aux langues tsiganes, africaines et surtout à la langue arabe (notamment à sa variante maghrébine, le darija). Voir à ce sujet, entre autres, Jean-Pierre Goudaillier, 2001, Comment tu tchatches!, Paris, Maisonneuve et Larose.

2. On entend ici par ce terme les écrivains qui traitent dans leurs œuvres le thème de l'«identité de banlieues de grandes villes européennes où les "origines" ethniques ou culturelles cèdent souvent la place à une conscience de marginalité » (Charles Bonn, 1990, p. 227). Un débat critique autour de cette étiquette, souvent jugée plus

3. Azouz Begag, 1995, Insel der Winde (L'Îlet-aux-Vents), tr. Regina, Keil, Innsbruck, Haymon; Azouz Begag, 1998, Der Junge vom Stadtrand (Le Gone du Chaâba), tr. Regina, Keil, Basilée, Nagel \& Kimche/ Baobab.

4. Mohamed Razane, 2008, Leve, overleve (Dit Violent), tr. Thomas, Lundbo, Minuskel

5. J'ai notamment traduit en italien Rachid Djaïdani, Mabrouck Rachedi, Samuel Benchetrit, le collectif « Qui fait la France? », ainsi que d'autres écrivains de la « postcolonie », comme Bessora. 6. En particulier Rachid Djaïdani, 2004, Mon $n e^{\oplus} f$, Paris, Seuil et Viscéral, 2007, Paris, Seuil. 
7. La plupart des études à ce sujet sont de matrice anglo-américaine ; cf. Susan Bassnett et Harish Trivedi (éd.), 1999, Post-colonial Translation. Theory and Practice, Londres, Routledge ; Sherry Simon et Paul St-Pierre (éd.), 2000, Changing the Terms. Translating in the Postcolonial Era, Ottawa, Ottawa University Press.

8. 2008, Il me sera difficile de venir te voir, La Roque d'Anthéron, Vent D'Ailleurs, p. 210.

9.

10. Louis-Jean Calvet, 2007, L'Argot, Paris, PUF (3e édition mise à jour), p. 93-94.

11. En appliquant au « français des cités » les fonctions principales du langage des ghettos noirs new-yorkais repérées par William Labov (The Study of Non-standard English, Washington, DC, National Council of Teachers of English, 1969), Jean-Pierre Goudaillier a identifié une nouvelle fonction "crypto-ludique ", pour indiquer que le phénomène de la crypticité est intrinsèquement lié au ludique. Voir le déjà cité Jean-Pierre Goudaillier, 2001, Comment tu tchatches!, Paris, Maisonneuve et Larose.

12. Pour l'explication complète de tous les phénomènes à l'œuvre dans la transformation de ces mots, je renvoie à Jean-Pierre Goudaillier, Comment tu tchatches !, cit.

13. Antoine Berman, 1999, La Traduction et la lettre ou l'auberge du lointain, Paris, Seuil.

14. Les réflexions traductologiques dans ce domaine (français > italien) se limitent, à ma connaissance, au seul secteur audiovisuel. Voir, par exemple, Chiara Elefante, « Arg. et pop., ces abréviations qui donnent les jetons aux traducteurs-dialoguistes », Meta: journal des traducteurs, vol. 49, n 1, avril 2004, p. 193-207.

15. Pour ne citer qu'un exemple, dans le Garzanti français/italien le terme «meuf » est traduit par donna (italien standard pour « femme »).

16. Voici quelques exemples de traduction de mots en argot ou en verlan tirés de ma traduction du roman Viscéral de Djaïdani: grana (fric); gabbio (taule); pulotto (keuf); tipa (meuf) ; strippare (flipper) ; ferro (caisse, dans le sens de voiture) ; sbroccato (ouf).

17. Pour ceux qui s'intéressent au français contemporain des cités, je signale notamment le Dictionnaire de la zone de Cobra le Cynique (<http://www.dictionnairedelazone.fr $>$ ), source inépuisable d'informations. Pour ce qui est de l'argot italien des jeunes, je signale le site Manuale di mitologia urbana (<http://www.bruttastoria.it>), ainsi que le dictionnaire de Renzo Ambrogio et Giovanni Casalegno, 2004, Scrostati gaggio ! Dizionario sto- rico dei linguaggi giovanili, UTET.

18. Antoine Berman, 1984, L'Épreuve de l'étranger. Culture et traduction dans l'Allemagne romantique, Paris, Gallimard.

19. Rachid Djaïdani, Viscerale. Un grido dalle banlieue, tr. Ilaria Vitali, Rome, Giulio Perrone Editore, 2009. Dans la version italienne, le titre est suivi d'un sous-titre explicatif (un grido dalle banlieue, littéralement « un cri des banlieues »), il s'agit d'un choix éditorial (les titres dépendent très fréquemment d'un choix de l'éditeur).

20. Cf. Fulvia Antonelli, 2009, « Classi pericolose a Bologna », Lo Straniero, octobre, p. 78-83.

21. Une stratégie de compensation similaire a été relevée par Katrien Lievois dans la traduction néerlandaise d'un ouvrage de Fouad Laroui. Cf. Katrien Lievois, 2008, «La traduction de l'allusion ironique dans La Fin tragique de Philomène Tralala de Fouad Laroui : quand l'autre n'est pas celui qu'on croit », Expressions maghrébines,

22. Francesco Paolo Alexandre Madonia, 2008, « Tradurre il plurilinguismo : francese mescidato e calchi dall'arabo in Naissance à l'aube di Driss Chraïbi ", in Laura, Restuccia et Giovanni Saverio, Santangelo (dir.), Écritures des migrations: passages et hospitalités, Palerme, Palumbo, p. 181-191.

23. Rachid Djaïdani, Mon ne ${ }^{\circledast}$, cit., p. 21.

24. Rachid Djaïdani, Ritratto di un ragazzo da buttare alle or tiche, cit., p. 14.

25. Rachid Djaïdani, Viscéral, cit., p. 154.

26. Rachid Djaïdani, Viscerale, cit., p. 128. 
27. Plusieurs auteurs participent en effet à des battles slam. Pour souligner cette nouvelle composante, l'éditeur Sarbacane a d'ailleurs créé un forum (<www.exprim-forum.com>) où l'on peut écouter et visionner des extraits «lus, mis en musique, slamés par les auteurs » et où on invite les lecteurs non seulement à envoyer leurs commentaires, mais aussi à participer à la battle slam en envoyant leurs créations artistiques. L'intuition de Sarbacane quant au côté sonore du texte " urbain » se lit également dans le choix de mettre en exergue de chaque roman une playlist, sorte de bande-son de l'ouvrage écrit.

28. Rachid Djaïdani, Viscéral, cit., p. 7.

29. Rachid Djaïdani, Mon ne ${ }^{\circledR}$, cit., p. 46.

30. Ibid., p. 81.

31. Rachid Djaïdani, Mon ne ${ }^{\circledR}$, cit., p. 82.

32. Lorenzo Jovanotti, (éd.), 1996, Potere alla parola. Antologia del rap italiano, Milan, Feltrinelli.

33. Il en a d'ailleurs tiré un film documentaire, intitulé Sur ma ligne, primé à la 8e Biennale des cinémas arabes en 2006.

34. 35.

36. Regina Keil-Sagawe, «La main de Fatima. Problématique du transfert culturel dans la traduction/réception de littérature maghrébine d'expression française en allemand », cit.

\section{AUTEUR}

\section{ILARIA VITALI}

ilaria.vitali@unibo.it

Ilaria Vitali est docteur en littératures française et comparée de l'Université Paris IV-Sorbonne et de l'Université de Bologne, où elle est actuellement chercheuse post-doctorat. Elle oriente son intérêt pour les écritures migrantes dans une double direction : la recherche et la traduction. Elle a dirigé des ouvrages collectifs portant sur les écritures de l'extrême contemporain (Intrangers, 2011 ; Expatriées, exilées, nomades..., 2010 ; Les Manifestes littéraires au tournant du XXI ${ }^{\mathrm{e}}$ siècle, 2010) et elle est l'auteur de nombreuses études sur les écrivains francophones « de la postcolonie », qu'elle a contribué à faire connaître en Italie à travers ses traductions. Dans les dernières années, elle s'est spécialisée plus particulièrement dans la traduction d'auteurs issus des banlieues françaises. Rachid Djaïdani, Mabrouck Rachedi, Samuel Benchetrit, Saphia Azzeddine ont été traduits, entre autres, par Ilaria Vitali. En 2011, elle a été accueillie comme traducteur en résidence, pour le mois de décembre, à la Prévôté de Bordeaux. 